OPEN ACCESS

Edited by:

Ronald M. Bukowski, Cleveland Clinic, United States

Reviewed by:

Andrew Thomas Lenis, Memorial Sloan Kettering Cancer

Center, United States Alfredo Berruti,

University of Brescia, Italy

*Correspondence: Guoyao Zhang

Guoyao_Zhang@163.com

${ }^{+}$These authors have contributed equally to this work and share first authorship

Specialty section: This article was submitted to Genitourinary Oncology, a section of the journal

Frontiers in Oncology

Received: 01 October 2020 Accepted: 22 March 2021 Published: 20 April 2021

Citation:

Zhang G, Shi W, Jia E, Zhang L, Han Y, Rodriguez $R$ and Ma T (2021) FOXO3A Expression in Upper Tract Urothelial Carcinoma.

Front. Oncol. 11:603681. doi: 10.3389/fonc.2021.603681

\section{FOX03A Expression in Upper Tract Urothelial Carcinoma}

\author{
Guoyao Zhang ${ }^{1 * t}$, Wanping Shi ${ }^{2 \dagger}$, Enzhao $\mathrm{Jia}^{3}$, Lei Zhang ${ }^{1}$, Yongsheng $\mathrm{Han}^{4}$, \\ Ronald Rodriguez ${ }^{5}$ and Tianjiang $\mathrm{Ma}^{1}$ \\ ${ }_{1}^{1}$ Department of Oncology, Luohe Central Hospital, The First Affiliated Hospital of Luohe Medical College, Luohe, China, \\ 2 Department of Oncology, The Second Affiliated Hospital, Chongqing Medical University, Chongqing, China, ${ }^{3}$ Department of \\ Pathology, Luohe Central Hospital, The First Affiliated Hospital of Luohe Medical College, Luohe, China, ${ }^{4}$ Department of \\ General Surgery, School of Medicine, Qinghai University, Xining, China, ${ }^{5}$ Department of Urology, University of Texas Health \\ Science Center San Antonio, San Antonio, TX, United States
}

Background: Epidemiological studies have reported various results regarding whether FOXO3A is related to various carcinomas. However, the prognostic significance of FOXO3A in upper tract urothelial carcinoma (UTUC) remains unclear. The purpose of this study was to validate the correlation between FOXO3A expression and oncological outcomes in UTUC.

Methods: The expression levels of FOXO3A in 107 UTUC patients were examined by immunohistochemistry $(\mathrm{IHC})$. We examined the prognostic role of FOXO3A by using the Cox proportional hazard model.

Results: The results indicated that FOXO3A expression was notably decreased in UTUC tissue compared with control tissue. Decreased expression of FOXO3A was also related to advanced pathologic stage $(P=0.026)$, lymph node metastasis $(P=0.040)$, lymphovascular invasion $(P<0.001)$, and adjuvant therapy $(P=0.048)$. In addition, UTUC patients with low FOXO3A expression had a significantly shorter survival time, including both overall survival (OS) [hazard ratio (HR) 2.382, $P=0.004$ ] and recurrencefree survival (RFS) (HR 2.385, $P=0.004)$, than those with high expression. Multivariate analyses showed that FOXO3A was a significant predictor for OS (HR 2.145, $P=0.014)$ and RFS (HR 2.227, $P=0.010$ ) in UTUC patients.

Conclusion: Our results indicate that FOXO3A may be involved in the recurrence of UTUC and that it has certain clinical value in the therapeutic targeting and prognostic evaluation of UTUC.

Keywords: FOXO3A, prognosis, overall survival, recurrence-free survival, upper tract urothelial carcinoma

\section{INTRODUCTION}

Urothelial carcinoma (UC) is the most common malignancy of the urinary tract. However, upper tract (renal pelvis and ureter) tumors account for only 5\% to $10 \%$ of all UCs, and almost $60 \%$ of upper tract UCs (UTUCs) are invasive at diagnosis $(1,2)$. Currently available prognostic tools that utilize clinical and pathological parameters are limited for UTUC. Through the analysis of 
biomarkers in pathological specimens, we may strengthen the risk stratification and guide better prognostic evaluations for a more effective therapeutic strategy (3-5).

Forkhead box O 3a (FOXO3A) belongs to the FOXO protein family and is located on human chromosome 6q21 (6). FOXO3A activity and stability can be regulated by post-translational modifications, including phosphorylation, acetylation, ubiquitination and glycosylation, aside from its well-validated modifications in transcription (7). It has been highlighted as an important transcriptional regulator of crucial proteins participating in DNA damage repair (8), cell cycle regulation (9), apoptosis (10), angiogenesis (11), and cellular stress response (12). The function and detailed molecular mechanisms of FOXO3A in tumor progression remain elusive. FOXO3A is downregulated and functions as a tumor suppressor in several types of tumors, including urothelial carcinoma. Downregulation of FOXO3A expression promotes tumor occurrence, metastasis, and progression in breast cancer (13), gastric carcinoma (14), pancreatic ductal adenocarcinoma (15), cervical carcinoma (16), clear cell renal cell carcinoma (17), and urothelial carcinoma (18).

In contrast, it plays a more complex supportive role in various types of malignancies. For example, in glioblastoma multiforme, overexpression of FOXO3A is positively correlated with tumor progression and predicts a poor survival outcome (19). Some studies have reported that activation of FOXO3A can lead to the elimination of cancer stem cells (20-22). FOXO3A also enhances the invasive ability of tumor cells by regulating matrix metalloproteinases in a number of tumor cell types $(23,24)$. However, whether FOXO3A serves as a useful biomarker in UTUC has not been reported.

Herein, we first detected FOXO3A by immunohistochemical analysis of UTUC patients and then investigated any potential association between FOXO3A and clinicopathologic parameters in patients with UTUC. Moreover, we examined the prognostic role of FOXO3A and aimed to build a predictive model for UTUC.

\section{METHODS}

\section{Patients}

The present study was carried out at Luohe Central Hospital, Luohe, China. Ethical approval for this study was obtained from the Institutional Ethics Committee of the Department of Pathology, Luohe Central Hospital, Luohe, China.

We collected UTUC samples between November 2004 and December 2015 from the archives for immunohistochemical and survival analysis. Finally, 107 UTUC patients who underwent surgery in the pathology department of Luohe Central Hospital were selected. The inclusion criteria were as follows: 1) patients with a diagnosis of UTUC who underwent radical nephroureterectomy and had clinicopathological data, 2) patients for whom concomitant bladder cancer was excluded on cystoscopy, and 3) patients without any other malignancies or severe chronic disease.
Regular follow-up had no standard protocol due to the retrospective nature of the study. Patients received clinical and radiological follow-up in accordance with final pathology, guidelines at that time, and physician judgement. Clinical data were extracted from medical records, including tumor number, pathological stage, histology grade, depth of invasion, lymph node metastasis (LNM) status, lymphovascular invasion (LVI), histological differentiation, adjuvant therapy and neoadjuvant chemotherapy. The clinical features of the UTUC patients are listed in Table 1.

\section{Immunohistochemistry}

IHC staining for FOXO3a was performed following the manufacturer's recommendations. Tissue section blocks were cut into $4-\mu \mathrm{m}$-thick slides, dewaxed and rehydrated. The antigen was recovered, and endogenous peroxidase activity was blocked. The slides were then incubated with anti-FOXO3a antibody (1:100 dilution; Abcam, Cambridge, UK) for 1 hour. The

TABLE 1 | Association between FOXO3A expression and UTUC clinicopathological parameters.

\begin{tabular}{|c|c|c|c|c|}
\hline Parameter & Overall & $\begin{array}{l}\text { FOXO3A (Low } \\
\text { expression) }\end{array}$ & $\begin{array}{l}\text { FOXO3A (High } \\
\text { expression) }\end{array}$ & $\begin{array}{c}P \text { - } \\
\text { value }\end{array}$ \\
\hline Patients (n) & 107 & 67 & 40 & \\
\hline \multicolumn{5}{|l|}{ Age, years } \\
\hline$<65$ & $49(45.8)$ & $30(44.8)$ & $19(47.5)$ & \multirow[t]{2}{*}{0.842} \\
\hline$\geq 65$ & $58(54.2)$ & 37 (55.2) & 21 (52.5) & \\
\hline \multicolumn{5}{|l|}{ Gender } \\
\hline Male & $71(66.4)$ & $42(62.7)$ & $29(72.5)$ & \multirow[t]{2}{*}{0.398} \\
\hline Female & 36 (33.6) & 25 (37.3) & $11(27.5)$ & \\
\hline \multicolumn{5}{|l|}{ Multifocality } \\
\hline Multifocal & 22 (20.6) & $12(17.9)$ & $10(25.0)$ & \multirow[t]{2}{*}{0.460} \\
\hline Single & 85 (79.4) & 55 (82.1) & $30(75.0)$ & \\
\hline \multicolumn{5}{|l|}{$\begin{array}{l}\text { Histologic } \\
\text { grade }\end{array}$} \\
\hline Low & 37 (34.6) & 27 (40.3) & $10(25.0)$ & \multirow[t]{2}{*}{0.142} \\
\hline High & $70(65.4)$ & $40(59.7)$ & $30(75.0)$ & \\
\hline \multicolumn{5}{|l|}{ pT stage } \\
\hline $\mathrm{Ta} / \mathrm{T} 1$ & $46(43.0)$ & 23 (34.3) & $23(57.5)$ & \multirow[t]{2}{*}{0.026} \\
\hline $\mathrm{T} 2 / \mathrm{T} 3 / \mathrm{T} 4$ & $61(57.0)$ & $44(65.7)$ & $17(42.5)$ & \\
\hline \multicolumn{5}{|l|}{ LNM } \\
\hline No & $66(61.7)$ & $36(53.7)$ & $30(75.0)$ & \multirow[t]{2}{*}{0.040} \\
\hline Yes & 41 (38.3) & 31 (46.3) & $10(25.0)$ & \\
\hline \multicolumn{5}{|l|}{ LVI } \\
\hline Absent & $53(49.5)$ & 23 (34.3) & $30(75.0)$ & \multirow[t]{2}{*}{$<0.001$} \\
\hline Present & 54 (50.5) & $44(65.7)$ & $10(25.0)$ & \\
\hline \multicolumn{5}{|l|}{$\begin{array}{l}\text { Histological } \\
\text { differentiation }\end{array}$} \\
\hline Pure urothelial & 89 (83.2) & 57 (85.1) & 32 (80.0) & \multirow[t]{2}{*}{0.595} \\
\hline $\begin{array}{l}\text { Variant } \\
\text { histology }^{\text {a }}\end{array}$ & $18(16.8)$ & $10(14.9)$ & $8(20.0)$ & \\
\hline \multicolumn{5}{|l|}{$\begin{array}{l}\text { Adjuvant } \\
\text { therapy }\end{array}$} \\
\hline Yes & 32 (29.9) & 25 (37.3) & $7(17.5)$ & \multirow[t]{2}{*}{0.048} \\
\hline No & 75 (70.1) & $42(62.7)$ & $33(82.5)$ & \\
\hline \multicolumn{5}{|l|}{$\begin{array}{l}\text { Neoadjuvant } \\
\text { chemotherapy }\end{array}$} \\
\hline Yes & $15(14.0)$ & $9(13.4)$ & $6(15.0)$ & \multirow[t]{2}{*}{1.000} \\
\hline No & $92(86.0)$ & $58(86.6)$ & $34(85.0)$ & \\
\hline
\end{tabular}

LNM, lymph node metastasis; LVI, lymphovascular invasion; $p T$, pathological tumor stage. ${ }^{a}$ Variant histology included micropapillary, plasmocytoid, sarcomatoid, and neuroendocrine types. 
presence of brown chromophores in the nucleus and cytoplasm of target cells indicated positive immunoreactivity. Finally, the slide was examined by optical microscopy at $400 \times$ magnification. Negative and positive controls were scored to optimize staining.

\section{Evaluation of the IHC Results}

FOXO3A-positive cells were assessed irrespective of the intensity of staining, and intracytoplasmic staining of FOXO3A was subsequently evaluated. The methodology applied by Tian et al. (16) was used to evaluate the FOXO3A score.

The percentage of cells positive for FOXO3A in the tumor stroma was recorded by two observers (TJM and LZ) as $0=$ no positive cells, $1=1-10 \%$ positive cells, $2=10-50 \%$ positive cells, $3=50-80 \%$ positive cells, and $4=>80 \%$ positive cells. The same score obtained by more than two observers was counted as the final score. Each specimen received a score of $0,1,2,3$ or 4 according to the intensity of FOXO3A staining. The product of the intensity score and stained area percentage was added and used as the total score. The final scores ranged from 0 to 8 and were designated low (0-4) or high (5-8).

\section{Statistical Analysis}

The analysis of FOXO3A IHC staining between tumor tissue and adjacent normal tissues was assessed by the t-test or chi-square using GraphPad Prism 8 software. When FOXO3a IHC was deep sectioned, the tumor component of three of the 43 tumor tissues was lost, so the FOXO3a expression of these tumors could not be assessed; ultimately, these three tumor tissues were excluded from the analyses involving the tumor component expression. All 40 pairs of UTUC and adjacent normal tissues were considered in the remaining analyses.

The SPSS 26.0 software suite (SPSS Inc., Chicago, IL, USA) was utilized for all statistical analyses, and statistical significance was considered at $P<0.05$. Associations between FOXO3A staining expression and clinicopathologic variables were estimated with Fisher's exact test. Overall survival (OS) was defined as tumor-related death, and recurrence-free survival (RFS) was defined as any local recurrence or distant recurrence, whichever occurred first. The difference in OS and RFS between the FOXO3A-high and FOXO3A-low groups was assessed in univariable and multivariable settings. Variables such as FOXO3A expression, pathological tumor stage, and histological differentiation were grouped into two groups: high expression vs low expression of FOXO3A, early stage (Ta-T1) vs late stage (T2-4), and pure urothelial vs variant histology.

\section{RESULTS}

\section{Expression of FOXO3A Protein Is Decreased in UTUC Patients}

Using the IHC staining method, the relative level of FOXO3A protein expression was assessed in 40 pairs of UTUC and adjacent normal tissues. Representative photographs of FOXO3A IHC are shown in Figures 1A-C. The scatter dot plot illustrated that the average immunoreactivity score of FOXO3A protein in 40 UTUC tissues was significantly downregulated compared with that of the 40 normal tissues $(3.08 \pm 0.43$ VS $4.30 \pm 0.44$ ) (Figure 1D, $P<$ 0.001). The expression of FOXO3A was lower in UTUC patients than in normal tissues (UTUC vs normal tissues: $13 / 40$ vs. $22 / 40$, $P<0.001)$.

\section{FOX03A Immunoexpression Is Associated With the Clinical Parameters of UTUC Patients}

The correlations of the relative FOXO3A expression level with clinical criteria are shown in Table 1. The results showed that low expression of FOXO3A was significantly associated with aggressive pathological stage $(P=0.026)$, lymphovascular invasion $(P<0.001)$, lymph node metastasis $(P=0.040)$, and adjuvant therapy $(P=0.048)$.

\section{Survival Analysis}

In the Kaplan-Meier analyses, patients with low FOXO3A expression had markedly worse OS $(P=0.003$; Figure 2A) and RFS $(P=0.003$; Figure 2B) than those with high FOXO3A expression. Furthermore, log rank tests demonstrated a significant difference between the 5-year OS (83.6\% in the high expression group vs. $60.7 \%$ in the low expression group) and RFS (83.5\% in the high expression group vs. $61.4 \%$ in the low expression group) rates in these two groups.

Univariate analyses determined that pathological stage (both $P=0.008$ ), lymph node metastasis (both $P<0.001$ ), lymphovascular invasion (both $P=0.028$ ), adjuvant therapy
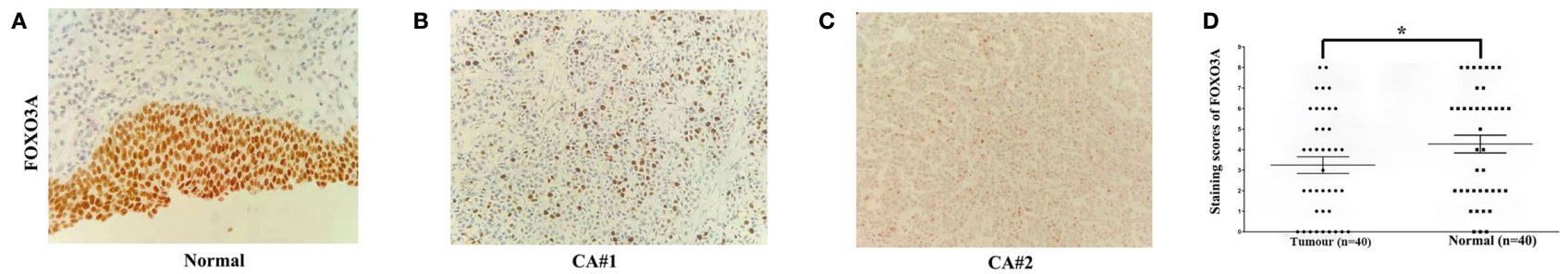

FIGURE 1 | FOXO3a expression in UTUC tissues determined by immunohistochemical staining (original magnification, 200x). (A-C) Representative FOXO3a expression in tumor and normal tissues, with positive expression located in the nucleus. (D) Scatter dot plot showing the staining score (mean \pm SEM) of FOXO3a in tumor and normal tissues using the paired t-test. ${ }^{*} \mathrm{P}<0.001$. 
A

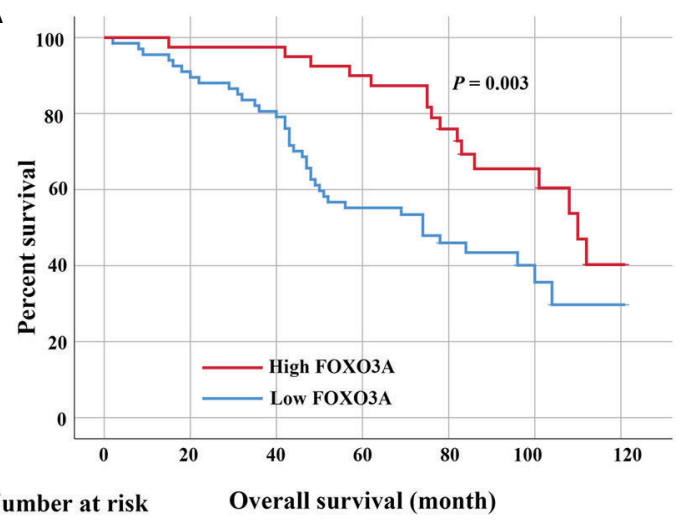

FOXO3A (Low expression)

$\begin{array}{lllllll}67 & 60 & 53 & 36 & 21 & 8 & 1\end{array}$

FOXO3A (High expression)

$\begin{array}{lllllll}40 & 39 & 39 & 36 & 24 & 13 & 1\end{array}$
B

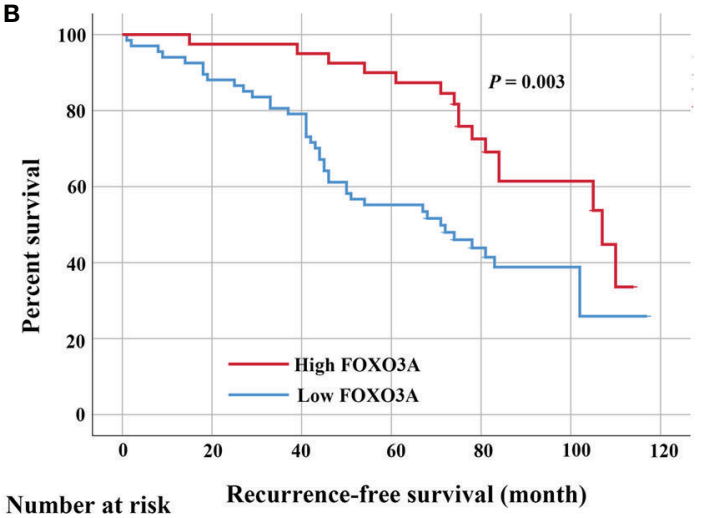

FOXO3A (Low expression)

$\begin{array}{lllllll}67 & 59 & 53 & 36 & 18 & 4 & 0\end{array}$

FOXO3A (High expression)

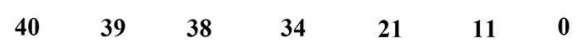

FIGURE 2 | (A) Kaplan-Meier curves for the 5-year OS rate of patients with UTUC and OS based on FOXO3a in UTUC patients. (B) Kaplan-Meier curves for the 5year RFS rate of patients with UTUC and RFS based on FOXO3a in UTUC patients. Error bar = SEM.

$(P=0.025$ and $P=0.015)$ and FOXO3a protein (both $P=0.004)$ were significantly correlated with both OS and RFS. Histologic grade $(P=0.049)$ was also associated with OS (Tables 2, 3). However, only pathological stage, lymph node metastasis and
FOXO3A protein expression were ultimately determined to be predictors of the OS $(P=0.031, P=0.006, P=0.014)$ and RFS $(P=0.022, P=0.004, P=0.010)$ of UTUC patients in the multivariate analyses (Tables 2,3 ).

TABLE 2 | Univariate and multivariable analyses assessing the association between predictor variables and overall survival mortality among 107 patients for UTUC.

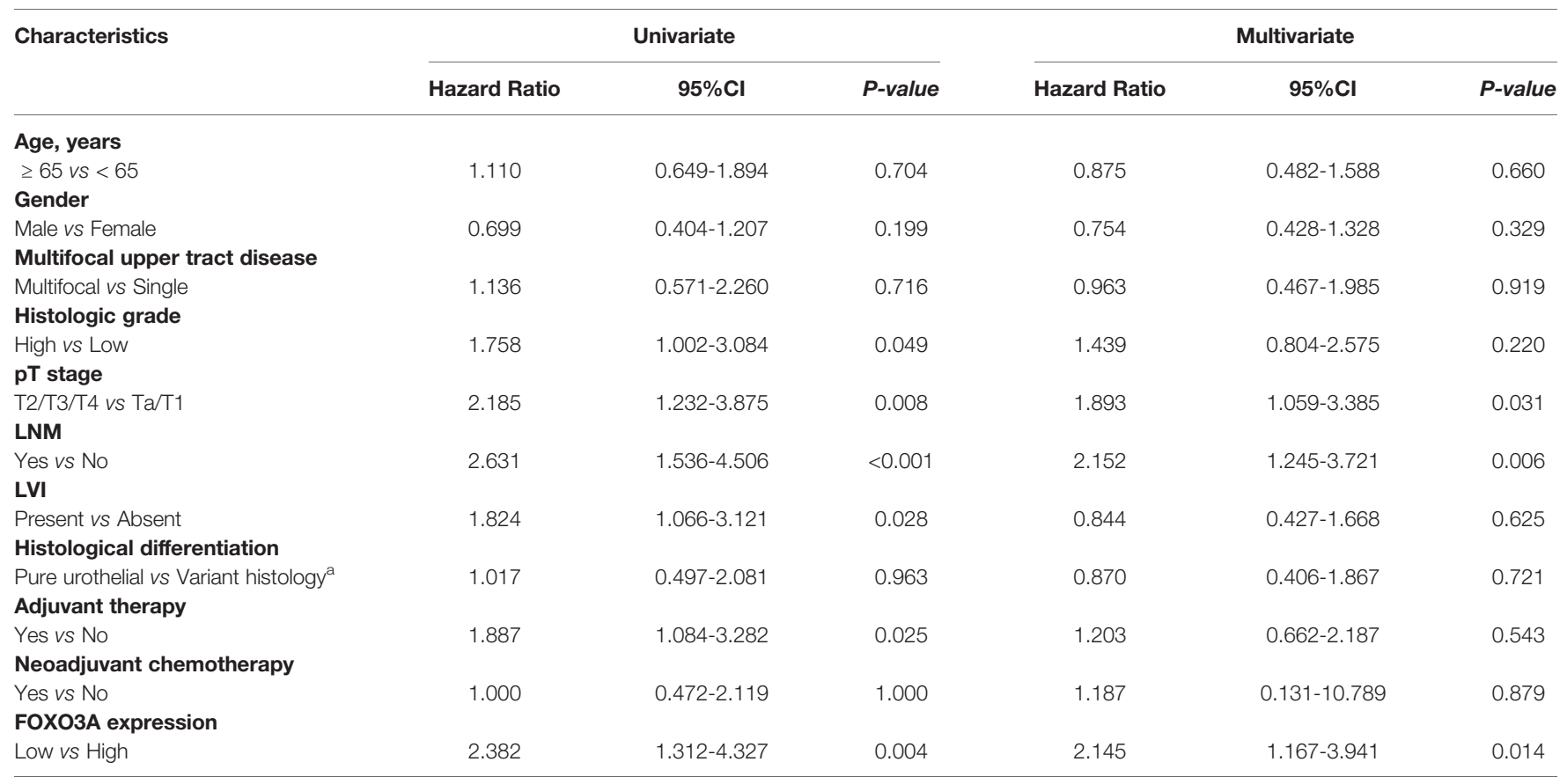

LNM, lymph node metastasis; LVI, lymphovascular invasion; $p T$, pathological tumor stage.

${ }^{a}$ Variant histology included micropapillary, plasmocytoid, sarcomatoid, and neuroendocrine types. 
TABLE 3 | Univariate and multivariable analyses assessing the association between predictor variables and recurrence among 107 patients for UTUC.

\begin{tabular}{|c|c|c|c|c|c|c|}
\hline \multirow[t]{2}{*}{ Characteristics } & \multicolumn{3}{|c|}{ Univariate } & \multicolumn{3}{|c|}{ Multivariate } \\
\hline & Hazard Ratio & $95 \% \mathrm{Cl}$ & $P$-value & Hazard Ratio & $95 \% \mathrm{Cl}$ & $P$-value \\
\hline \multicolumn{7}{|l|}{ Age, years } \\
\hline$\geq 65 v s<65$ & 1.100 & $0.645-1.877$ & 0.726 & 0.876 & $0.490-1.567$ & 0.655 \\
\hline \multicolumn{7}{|l|}{ Gender } \\
\hline Male vs Female & 0.671 & $0.386-1.167$ & 0.158 & 0.713 & $0.403-1.263$ & 0.247 \\
\hline \multicolumn{7}{|l|}{ Multifocal upper tract disease } \\
\hline Multifocal vs Single & 1.189 & $0.598-2.367$ & 0.621 & 0.971 & $0.470-2.005$ & 0.913 \\
\hline \multicolumn{7}{|l|}{ Histologic grade } \\
\hline High vs Low & 1.708 & $0.975-2.993$ & 0.062 & 1.298 & $0.715-2.356$ & 0.391 \\
\hline \multicolumn{7}{|l|}{ pT stage } \\
\hline $\mathrm{T} 2 / \mathrm{T} 3 / \mathrm{T} 4$ vs $\mathrm{Ta} / \mathrm{T} 1$ & 2.181 & $1.229-3.871$ & 0.008 & 1.971 & $1.102-3.527$ & 0.022 \\
\hline \multicolumn{7}{|l|}{ LNM } \\
\hline Yes vs No & 2.708 & $1.580-4.643$ & $<0.001$ & 2.257 & $1.306-3.900$ & 0.004 \\
\hline \multicolumn{7}{|l|}{ LVI } \\
\hline Present vs Absent & 1.834 & $1.069-3.144$ & 0.028 & 0.844 & $0.426-1.670$ & 0.626 \\
\hline \multicolumn{7}{|l|}{ Histological differentiation } \\
\hline Pure urothelial vs Variant histology ${ }^{a}$ & 1.055 & $0.515-2.165$ & 0.883 & 0.860 & $0.397-1.865$ & 0.703 \\
\hline \multicolumn{7}{|l|}{ Adjuvant therapy } \\
\hline Yes vs No & 1.991 & $1.142-3.471$ & 0.015 & 1.402 & $0.779-2.521$ & 0.259 \\
\hline \multicolumn{7}{|l|}{ Neoadjuvant chemotherapy } \\
\hline Yes vs No & 1.039 & $0.488-2.209$ & 0.922 & 1.144 & $0.125-10.491$ & 0.906 \\
\hline \multicolumn{7}{|l|}{ FOXO3A expression } \\
\hline Low vs High & 2.385 & $1.314-4.329$ & 0.004 & 2.227 & $1.209-4.101$ & 0.010 \\
\hline
\end{tabular}

LNM, lymph node metastasis; LVI, lymphovascular invasion; pT, pathological tumor stage.

a Variant histology included micropapillary, plasmocytoid, sarcomatoid, and neuroendocrine types.

\section{DISCUSSION}

Most recent studies have investigated whether FOXO3A plays a key role in UC. Shiota et al. (18) showed that FOXO3A inhibits UC invasiveness via Twist1, YB-1, and E-cadherin regulation. Zhuo et al. (25) reported that upregulation of CSTP1 expression suppresses IL6 expression by regulating the Akt/FoxO3a signaling pathway in UC. Zhu et al. (26) showed that ATG7 overexpression promoted autophagic removal of FOXO3A in bladder carcinoma cells. Nevertheless, little is known about the prognostic role and clinicopathologic implications of FOXO3A in UTUC.

The results of our study reveal the following: (1) low FOXO3A expression predicts unfavorable survival and recurrence rates in UTUC patients; (2) FOXO3A expression is negatively associated with pathological stage, lymph node metastasis status, lymphovascular invasion, and adjuvant therapy in UTUC patients; and (3) FOXO3A expression is lower in UTUC tissue than in normal tissue. These findings indicate that FOXO3A is a prognostic factor for UTUC and that adjuvant therapy may be helpful in the high-risk subgroup of UTUC patients.

The biological mechanism of FOXO3A also illustrates its key role in the pathogenesis of UTUC. FOXO3A is part of a subfamily of winged-helix transcription factors, and its activity can be regulated by PI3K/AKT signaling (6). FOXO3A has been identified as a tumor suppressor because of its ability to promote cell cycle arrest (27) and DNA damage repair (28) and to inhibit tumor cell properties and tumorigenesis (22). Interestingly, several potential substrates for correlations between FOXO3A and tumor metastases have been described in previous studies $(15,29)$. In addition, FOXO3A has been implicated in epithelial mesenchymal transition, an important process during metastasis, and downregulation of FOXO3A promotes tumor cell migration and invasion (30-32).

This study is the first to evaluate the associations between FOXO3A expression and clinicopathological features and prognostic factors in UTUC by IHC. FOXO3A can be considered an anti-oncogene, and overexpression or pharmacological activation of FOXO3A inhibits tumor progression and improves prognosis. A previous study confirmed that downregulation of FOXO3a expression is associated with poor prognosis in bladder carcinoma patients by RT-PCR. Based on these data, we suggest that FOXO3A expression in UTUC tends to indicate a good prognosis.

However, there are still limitations to the study. 1) UTUC is rare, and the sample size was relatively small. 2) Determination of FOXO3A status is limited to IHC detection of the protein without integrated methodology or a scoring system. 3) The detailed molecular mechanism of FOXO3A in UTUC remains unknown. 4) Because this is a retrospective single-center study, potential bias exists and cannot avoid confounding factors and the absence of a standard for follow-up assessment. Therefore, larger patient groups are needed to further investigate the role of FOXO3A in UTUC and help us to better understand the molecular events involved in the pathogenesis of UTUC.

There is a complex crosstalk between FOXO3a/AKT signaling pathway and PD-L1 involved in tumorigenesis $(26,33)$. PD-L1 is a critical regulator in UC development and the level of functional PD-L1 plays a vital role in the effective immunotherapeutic treatments for UC $(34,35)$. Therefore, further exploration of the relationship between FOXO3a and PD-1 may have important implications for UTUC immunotherapy. FOXO3a activity is directly regulated by some miRNAs in UC $(26,36)$. This suggests that finding or synthesizing new chemotherapeutic 
drugs targeting these miRNAs may also be a promising strategy for the treatment of UTUC.

In conclusion, our results showed that FOXO3A may be a potential biomarker for determining UTUC diagnosis and prognosis and may serve as a tumor-suppressing gene. Moreover, clarification of the underlying molecular mechanisms of FOXO3A in UTUC progression could aid in the development of targeted therapies for UTUC patients.

\section{DATA AVAILABILITY STATEMENT}

The original contributions presented in the study are included in the article/supplementary material. Further inquiries can be directed to the corresponding author.

\section{REFERENCES}

1. Tay LJ, Chatterton K, Colemeadow J, Nair R, Bultitude M, Thomas K. Improving management of upper tract urothelial carcinoma. BJU Int (2020) 126:5-6. doi: 10.1111/bju.15068

2. Roupret M, Babjuk M, Burger M, Capoun O, Cohen D, Comperat EM, et al. European Association of Urology Guidelines on Upper Urinary Tract Urothelial Carcinoma: 2020 Update. Eur Urol (2021) 79:62-79. doi: 10.1016/j.eururo.2020.05.042

3. Roberts JL, Ghali F, Aganovic L, Bechis S, Healy K, Rivera-Sanfeliz G, et al. Diagnosis, management, and follow-up of upper tract urothelial carcinoma: an interdisciplinary collaboration between urology and radiology. Abdom Radiol (NY) (2019) 44:3893-905. doi: 10.1007/s00261-019-02293-9

4. Hassler MR, Bray F, Catto JWF, Grollman AP, Hartmann A, Margulis V, et al. Molecular Characterization of Upper Tract Urothelial Carcinoma in the Era of Next-generation Sequencing: A Systematic Review of the Current Literature. Eur Urol (2020) 78:209-20. doi: 10.1016/j.eururo.2020.05.039

5. Mori K, Janisch F, Mostafaei H, Lysenko I, Kimura S, Egawa S, et al. Prognostic value of preoperative blood-based biomarkers in upper tract urothelial carcinoma treated with nephroureterectomy: A systematic review and meta-analysis. Urol Oncol (2020) 38:315-33. doi: 10.1016/ j.urolonc.2020.01.015

6. Alvarez B, Martinez AC, Burgering BM, Carrera AC. Forkhead transcription factors contribute to execution of the mitotic programme in mammals. Nature (2001) 413:744-7. doi: 10.1038/35099574

7. Kim CG, Lee H, Gupta N, Ramachandran S, Kaushik I, Srivastava S, et al. Role of Forkhead Box Class O proteins in cancer progression and metastasis. Semin Cancer Biol (2018) 50:142-51. doi: 10.1016/j.semcancer.2017.07.007

8. White RR, Maslov AY, Lee M, Wilner SE, Levy M, Vijg J. FOXO3a acts to suppress DNA double-strand break-induced mutations. Aging Cell (2020) 19: e13184. doi: 10.1111/acel.13184

9. Usami M, Kikuchi S, Takada K, Ono M, Sugama Y, Arihara Y, et al. FOXO3a Activation by HDAC Class IIa Inhibition Induces Cell Cycle Arrest in Pancreatic Cancer Cells. Pancreas (2020) 49:135-42. doi: 10.1097/ MPA.0000000000001462

10. Lee N, Tilija Pun N, Jang WJ, Bae JW, Jeong CH. Pitavastatin induces apoptosis in oral squamous cell carcinoma through activation of FOXO3a. J Cell Mol Med (2020) 24:7055-66. doi: 10.1111/jcmm.15389

11. Sun Z, Li M, Bai L, Fu J, Lu J, Wu M, et al. Arsenic trioxide inhibits angiogenesis in vitro and in vivo by upregulating FoxO3a. Toxicol Lett (2019) 315:1-8. doi: 10.1016/j.toxlet.2019.08.009

12. Fasano C, Disciglio V, Bertora S, Lepore Signorile M, Simone C. FOXO3a from the Nucleus to the Mitochondria: A Round Trip in Cellular Stress Response. Cells (2019) 8:1110. doi: 10.3390/cells8091110

13. Pellegrino M, Rizza P, Dona A, Nigro A, Ricci E, Fiorillo M, et al. FoxO3a as a Positive Prognostic Marker and a Therapeutic Target in Tamoxifen-Resistant Breast Cancer. Cancers (Basel) (2019) 11:1858. doi: 10.3390/cancers11121858

\section{ETHICS STATEMENT}

The studies involving human participants were reviewed and approved by The Institutional Ethics Committee of Luohe Central Hospital, Luohe, China. The patients/participants provided their written informed consent to participate in this study.

\section{AUTHOR CONTRIBUTIONS}

GZ, EJ, and LZ: writing and figures. GZ, WS, YH, RR, and TM: concept and proof reading. All authors contributed to the article and approved the submitted version.

14. Hu C, Ni Z, Li BS, Yong X, Yang X, Zhang JW, et al. hTERT promotes the invasion of gastric cancer cells by enhancing FOXO3a ubiquitination and subsequent ITGB1 upregulation. Gut (2017) 66:31-42. doi: 10.1136/gutjnl2015-309322

15. Li J, Yang R, Dong Y, Chen M, Wang Y, Wang G. Knockdown of FOXO3a induces epithelial-mesenchymal transition and promotes metastasis of pancreatic ductal adenocarcinoma by activation of the beta-catenin/TCF4 pathway through SPRY2. J Exp Clin Cancer Res (2019) 38:38. doi: 10.1186/ s13046-019-1046-X

16. Tian Y, Qi P, Hu X. Downregulated FOXO3a Associates With Poor Prognosis and Promotes Cell Invasion and Migration via WNT/beta-catenin Signaling in Cervical Carcinoma. Front Oncol (2020) 10:903. doi: 10.3389/fonc.2020.00903

17. Ni D, Ma X, Li HZ, Gao Y, Li XT, Zhang Y, et al. Downregulation of FOXO3a promotes tumor metastasis and is associated with metastasis-free survival of patients with clear cell renal cell carcinoma. Clin Cancer Res (2014) 20:177990. doi: 10.1158/1078-0432.CCR-13-1687

18. Shiota M, Song Y, Yokomizo A, Kiyoshima K, Tada Y, Uchino H, et al. Foxo3a suppression of urothelial cancer invasiveness through Twist1, Y-box-binding protein 1, and E-cadherin regulation. Clin Cancer Res (2010) 16:5654-63. doi: 10.1158/1078-0432.CCR-10-0376

19. Qian Z, Ren L, Wu D, Yang X, Zhou Z, Nie Q, et al. Overexpression of FoxO3a is associated with glioblastoma progression and predicts poor patient prognosis. Int J Cancer (2017) 140:2792-804. doi: 10.1002/ijc.30690

20. Chiu CF, Chang YW, Kuo KT, Shen YS, Liu CY, Yu YH, et al. NF-kappaBdriven suppression of FOXO3a contributes to EGFR mutation-independent gefitinib resistance. Proc Natl Acad Sci U S A (2016) 113:E2526-35. doi: $10.1073 /$ pnas. 1522612113

21. Sato A, Okada M, Shibuya K, Watanabe E, Seino S, Narita Y, et al. Pivotal role for ROS activation of p38 MAPK in the control of differentiation and tumorinitiating capacity of glioma-initiating cells. Stem Cell Res (2014) 12:119-31. doi: 10.1016/j.scr.2013.09.012

22. Liu H, Song Y, Qiu H, Liu Y, Luo K, Yi Y, et al. Downregulation of FOXO3a by DNMT1 promotes breast cancer stem cell properties and tumorigenesis. Cell Death Differ (2020) 27:966-83. doi: 10.1038/s41418-019-0389-3

23. Storz P, Doppler H, Copland JA, Simpson KJ, Toker A. FOXO3a promotes tumor cell invasion through the induction of matrix metalloproteinases. Mol Cell Biol (2009) 29:4906-17. doi: 10.1128/MCB.00077-09

24. Xu K, Pei H, Zhang Z, Dong S, Fu RJ, Wang WM, et al. FoxO3a mediates glioma cell invasion by regulating MMP9 expression. Oncol Rep (2016) 36:3044-50. doi: 10.3892/or.2016.5087

25. Zhuo D, Wu Y, Luo J, Deng L, Niu X. CSTP1 inhibits IL-6 expression through targeting Akt/FoxO3a signaling pathway in bladder cancer cells. Exp Cell Res (2019) 380:80-9. doi: 10.1016/j.yexcr.2019.04.019

26. Zhu J, Li Y, Luo Y, Xu J, Liufu H, Tian Z, et al. A Feedback Loop Formed by ATG7/Autophagy, FOXO3a/miR-145 and PD-L1 Regulates Stem-Like Properties and Invasion in Human Bladder Cancer. Cancers (Basel) (2019) 11:349. doi: 10.3390/cancers 11030349 
27. Liu Z, Shi Z, Lin J, Zhao S, Hao M, Xu J, et al. Piperlongumine-induced nuclear translocation of the FOXO3A transcription factor triggers BIMmediated apoptosis in cancer cells. Biochem Pharmacol (2019) 163:101-10. doi: 10.1016/j.bcp.2019.02.012

28. Zhou Y, Chen E, Tang Y, Mao J, Shen J, Zheng X, et al. miR-223 overexpression inhibits doxorubicin-induced autophagy by targeting FOXO3a and reverses chemoresistance in hepatocellular carcinoma cells. Cell Death Dis (2019) 10:843. doi: 10.1038/s41419-019-2053-8

29. Li R, Quan Y, Xia W. SIRT3 inhibits prostate cancer metastasis through regulation of FOXO3A by suppressing Wnt/beta-catenin pathway. Exp Cell Res (2018) 364:143-51. doi: 10.1016/j.yexcr.2018.01.036

30. Liu H, Yin J, Wang H, Jiang G, Deng M, Zhang G, et al. FOXO3a modulates WNT/beta-catenin signaling and suppresses epithelial-to-mesenchymal transition in prostate cancer cells. Cell Signal (2015) 27:510-8. doi: 10.1016/ j.cellsig.2015.01.001

31. Luo M, Wu C, Guo E, Peng S, Zhang L, Sun W, et al. FOXO3a knockdown promotes radioresistance in nasopharyngeal carcinoma by inducing epithelial-mesenchymal transition and the Wnt/beta-catenin signaling pathway. Cancer Lett (2019) 455:26-35. doi: 10.1016/j.canlet.2019.04.019

32. Bakir B, Chiarella AM, Pitarresi JR, Rustgi AK. EMT, MET, Plasticity, and Tumor Metastasis. Trends Cell Biol (2020) 30:764-76. doi: 10.1016/ j.tcb.2020.07.003

33. Chen HN, Liang KH, Lai JK, Lan CH, Liao MY, Hung SH, et al. EpCAM Signaling Promotes Tumor Progression and Protein Stability of PD-L1 through the EGFR Pathway. Cancer Res (2020) 80:5035-50. doi: 10.1158/ 0008-5472.CAN-20-1264

34. Zhou TC, Sankin AI, Porcelli SA, Perlin DS, Schoenberg MP, Zang X. A review of the PD-1/PD-L1 checkpoint in bladder cancer: From mediator of immune escape to target for treatment. Urol Oncol (2017) 35:14-20. doi: 10.1016/j.urolonc.2016.10.004

35. Powles T, Eder JP, Fine GD, Braiteh FS, Loriot Y, Cruz C, et al. MPDL3280A (anti-PD-L1) treatment leads to clinical activity in metastatic bladder cancer. Nature (2014) 515:558-62. doi: 10.1038/nature13904

36. Chang Y, Jin H, Li H, Ma J, Zheng Z, Sun B, et al. MiRNA-516a promotes bladder cancer metastasis by inhibiting MMP9 protein degradation via the AKT/ FOXO3A/SMURF1 axis. Clin Transl Med (2020) 10:e263. doi: 10.1002/ctm2.263s

Conflict of Interest: The authors declare that the research was conducted in the absence of any commercial or financial relationships that could be construed as a potential conflict of interest.

Copyright (C) 2021 Zhang, Shi, Jia, Zhang, Han, Rodriguez and Ma. This is an openaccess article distributed under the terms of the Creative Commons Attribution License (CC BY). The use, distribution or reproduction in other forums is permitted, provided the original author(s) and the copyright owner(s) are credited and that the original publication in this journal is cited, in accordance with accepted academic practice. No use, distribution or reproduction is permitted which does not comply with these terms. 